\title{
SURGERY ON LINKS CONTAINING A CABLE SUBLINK
}

\author{
BRADD EVANS CLARK
}

\begin{abstract}
In this paper it will be shown that there is an upper bound on the genus of any manifold obtained by Dehn surgery on a given torus link. It is also demonstrated that if $L$ is a link in $S^{3}$ with a cable sublink about the knot $k$, and surgery on at least two components of the sublink cannot be replaced by surgery on $k$, then the manifold resulting from surgery on $L$ cannot be simply connected.
\end{abstract}

1. Preliminaries. People have long been looking for a counterexample to the Poincaré conjecture by performing surgery on various knots. When Lickorish in [4] demonstrated that all compact connected orientable 3-manifolds without boundary could be represented as a result of surgery on a link in $S^{3}$, the search was extended to manifolds obtained by surgery on various links. Some of the first knots to be considered include torus and cable knots in [3] and [7], respectively. Torus links were handled in [2]. This paper will consider an arbitrary link which has a cable sublink.

If $X$ is a point set, we shall use $\operatorname{int}(X)$ for the interior of $X, \operatorname{cl}(X)$ for the closure of $X$, and $\partial X$ for the boundary of $X$. If $T$ is a solid torus and $D^{2}$ is a meridional disk of $T$, we can use $m=\partial D^{2}$ along with any transverse simple closed curve $l$ on $\partial T$ to form a coordinate system. By a $(p, q)$ curve we mean any simple closed curve on $\partial T$ which is isotopic to the "standard" curve which intersects $m p$-times and $l q$-times. The genus of a manifold is defined to be the minimal genus of a Heegaard splitting of the manifold. Let $X$ be a polyhedron contained in the P.L. $n$-manifold $M . N(X) \subset M$ is called a regular neighborhood of $X$ in $M$ if $X \subset N(X)$ and $N(X)$ is an n-manifold which can be simplicially collapsed to $X$.

This paper deals with piecewise linear topology. As such, all manifolds are considered to be simplicial and maps to be piecewise linear. This will be assumed as additional hypotheses throughout the paper.

2. Surgery on torus links. In considering Dehn surgery on a torus link, we shall find it convenient to use a somewhat different coordinate system from the usual one to describe the surgery.

Let $T$ be a solid torus and $L$ a torus link with $L \subset \partial T$. If $J_{i}$ is the $i$ th component of $L$ we can find a regular neighborhood $T_{i}$ of $J_{i}$ such that $T_{i} \cap T$ is an annulus. We can also find regular neighborhoods of $J_{i}$ and $J_{j}, T_{i}$ and $T_{j}$, such that $T_{i} \cap T_{j}=\varnothing$ if $i \neq j$. We shall use the usual meridian in our

Received by the editors December 19, 1977 and, in revised form, April 9, 1978.

AMS (MOS) subject classifications (1970). Primary 55A25.

Key words and phrases. Link, surgery, 3-manifold. 
coordinate system, but will use one boundary component of the annulus of sewing between $T$ and $T_{i}$ as the longitude. Since this is not the usual longitude, we can obtain strange looking results, such as $(2,-5)$ surgery on the trefoil being a homology sphere.

We also note that $T \cup T_{i}$ is a solid torus after a $\left(p_{i}, q_{i}\right)$ surgery on $J_{i}$ precisely when $q_{i}= \pm 1$. Since a $(-p,-q)$ curve is equivalent for surgery purposes to a $(p, q)$ curve, we shall assume that $q \geqslant 0$.

THEOREM 2.1. If $M$ is a 3-manifold obtained by surgery along a torus link with $n$ components, then $M$ has genus at most $n+1$.

Proof. Let $T$ be an unknotted solid torus in $S^{3}$. We split $\partial T$ into $2 n$ annuli $\left\{A_{1}, B_{1}, A_{2}, B_{2}, \ldots, A_{n}, B_{n}\right\}$ each following an $(x, y)$ curve on $\partial T$. Let $\partial T \times$ $I$ be a collar attached to $T$ with $\partial T=\partial T \times\{0\}$.

We can think of $M$ as being obtained by surgery on $A_{i} \times\left[\frac{1}{2}, 1\right]$ where $A_{i} \times I \subset \partial T \times I$. Let $D_{i}$ be a disk in $A_{i}$ with the property that $\operatorname{cl}\left(A_{i}-D_{i}\right)$ is a disk. Let $\alpha_{i}$ and $\beta_{i}$ be arcs in $\partial A_{i}$ with the property that

$$
\partial A_{i} \cap\left(\operatorname{cl}\left(A_{i}-D_{i}\right)\right)=\alpha_{i} \cup \beta_{i} \text {. }
$$

Let $T^{\prime}=\operatorname{cl}(M-(T \cup(\partial T \times I)))$. $T^{\prime}$ is a solid torus. Let $N_{i}=h\left(A_{i} \times\right.$ $\left.\left[\frac{1}{2}, 1\right]\right) \subset M$ be the image of $A \times\left[\frac{1}{2}, 1\right]$ in $M$ after surgery is performed on the torus link.

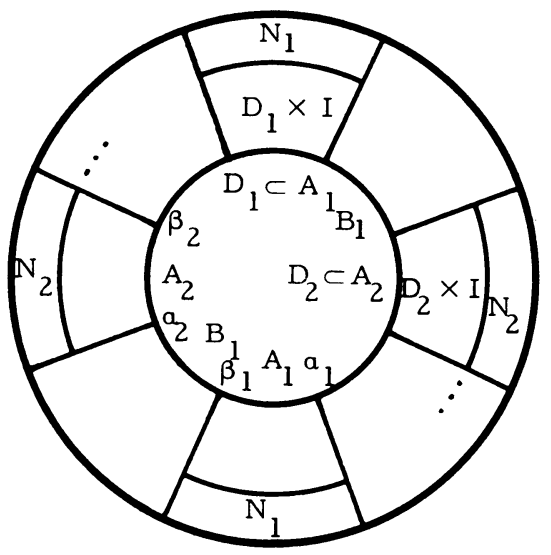

FIGURE 1

Since $D_{i} \times\left[0, \frac{1}{2}\right] \subset \partial T \times I$ is a 3-cell and $\left(D_{i} \times\left[0, \frac{1}{2}\right]\right) \cap T$ and $\left(D_{i} \times\left[0, \frac{1}{2}\right]\right) \cap N_{i}$ are disks, we have that $K=T \cup{ }_{i=1}^{n}\left[\left(D_{i} \times I\right) \cup N_{i}\right]$ is a cube with $n+1$ handles. Clearly $T^{\prime \prime}=T^{\prime} \cup\left(B_{i} \times I\right)$ is a solid torus where $B_{i} \times I \subset \partial T \times I$. Sifce $\alpha_{i} \times \S I$ and $\beta_{i} \times I$ are disks on $\partial T^{\prime \prime}$ where $\alpha_{i} \times I$ and $\beta_{i} \times I$ are contained in $\partial T \times I$, we have that $\operatorname{cl}\left(A_{i}-D_{i}\right) \times I \subset \partial T \times I$ is a handle added to $T^{\prime \prime}$. So $M-$ int $K$ is also a cube with $n+1$ handles. 
3. Surgery on links with a cable sublink. Suppose that $L$ is a link in $S^{3}$.

Definition. $L$ is said to contain a cable sublink if it contains a finite collection of simple closed curves $\left\{J_{1}, \ldots, J_{n}\right\}$ such that each $J_{i}$ is an $(x, y)$ cable about a specific knot $k \subset S^{3}$, and there exists a regular neighborhood of $k, N(k)$, such that $N(k) \cap L=\bigcup_{i=1}^{n} J_{i}$.

Assume that $M$ is formed by a $\left(p_{i}, q_{i}\right)$ surgery on some component $J_{i}$ of the cable sublink. Let $T$ be a regular neighborhood of $k$ and $T_{i}$ a regular neighborhood of $J_{i}$ such that $T \cap T_{i}$ is the annulus $A_{i}$. If $h\left(T_{i}\right)$ is the image of $T_{i}$ in $M$ after surgery on $L$, then we let $M_{i}=h\left(T_{i}\right) \cup T$.

A matrix representation of the self homeomorphism of $T_{i}$ relative to the boundary can be found, say

$$
\left[\begin{array}{cc}
n_{i} & m_{i} \\
p_{i} & q_{i}
\end{array}\right] \quad \text { where } \operatorname{det}\left[\begin{array}{cc}
n_{i} & m_{i} \\
p_{i} & q_{i}
\end{array}\right]=1
$$

If we consider the inverse of this matrix operating on $l$, we find that a $\left(p_{i}, q_{i}\right)$ surgery on $T_{i}$ results in a submanifold $M_{i}$ being formed by sewing an annulus following an $(x, y)$ curve on $\partial T$ to an annulus following a $\left(q_{i},-m_{i}\right)$ curve on $\partial T_{i}$. If $q_{i}=1$, then $M_{i}$ is a solid torus. Hence surgery on $J_{i}$ will, in this case, be the same as if surgery had been performed on $k$. We say that surgery on $J_{i}$ could have been replaced by surgery on $k$ whenever $M_{i}$ is a solid torus. Since surgery on $J_{i}$ could have been replaced by surgery on $k$ if $x=1$, we shall assume $x>1$.

THEOREM 3.1. $M_{i}$ is irreducible and has incompressible boundary if $q_{i}>1$. Furthermore, $M$ can be simply connected only if $m_{i} \equiv \pm x \bmod q_{i}$ and $y \equiv$ $\pm q_{i} \bmod x$.

The proof of this theorem was given in [1]

Proposition 3.2. Let $M$ be an irreducible 3-manifold with an incompressible torus boundary. Let $T$ be a solid torus. If $T$ is attached to $M$ by sewing $a$ nonseparating, nonmeridional annulus of $\partial T$ to a nonseparating annulus of $M$, the result is a 3-manifold with incompressible boundary.

Proof. Suppose the proposition is false. Then we can find a disk $D$ with $\partial D \subset \partial(M \cup T)$ and $\partial D$ a nontrivial element of $\pi_{1}(\partial(M \cup T))$. Let $A$ be the annulus of sewing between $M$ and $T$. We put $D$ in general position with respect to $A$ and get that $D \cap A$ is a finite collection of simple closed curves and arcs whose endpoints lie on $\partial D$.

If $D \cap A=\varnothing$, then $D \subset M$ or $D \subset T$. If $D \subset M$, we have that $\partial D$ is trivial in $\partial M$ since $M$ has incompressible boundary. But since $\partial D \cap A=\varnothing$, this means that $\partial D$ is a trivial element of $\pi_{1}(\partial(M \cup T))$. This is a contradiction.

If $D \subset T$ and $\partial D$ bounds a disk in $\partial T$, the above argument also holds. If $D$ is a meridional disk of $T$, then $A$ must be a meridional annulus which is also a 
contradiction. Therefore, $D \cap A \neq \varnothing$, and removing the intersections by isotopy of $D$ will be a contradiction.

Let $\left\{J_{1}, \ldots, J_{n}\right\}$ be the collection of simple closed curves in $D \cap A$. We shall call the curve $J_{i}$ innermost on $D$ if the subdisk $D_{i}$ bounded by $J_{i}$ has the property that $D_{i} \cap(D \cap A)=J_{i}$. Thus we have that either $D_{i} \subset M$ or $D_{i} \subset T$. If $D_{i} \subset M$ we have that there exists a disk $D_{i}^{\prime} \subset \partial M$ with $\partial D_{i}^{\prime}=\partial D_{i}$ $=J_{i}$. Since $M$ is irreducible, $D_{i} \cup D_{i}^{\prime}$ bounds a 3-cell which can be used to isotope $D_{i}$ to $D_{i}^{\prime}$. If $D_{i}^{\prime} \subset A$ we can use the product structure of $M \cup T$ at $A$ to push $D_{i}$ past $D_{i}^{\prime}$ and remove at least one simple closed curve of $D \cap A$. If $D_{i}^{\prime} \not \subset A$, we have that $A$ separates the boundary of $M$ which is also a contradiction.

If $D_{i} \subset T, D_{i}$ cannot be a meridional disk of $T$ since then $A$ would have to be a meridional annulus. Thus $D_{i}$ must have a companion disk $D_{i}^{\prime} \subset \partial T$ with $\partial D_{i}=\partial D_{i}=J_{i}$. The same argument as above either gives us a contradiction or allows us to isotope $D$ to a new disk in $M \cup T$ which has fewer simple closed curves in $D \cap A$.

We may now assume that $D \cap A$ has only arcs with endpoints on $\partial D$ for elements. We call a subdisk $D_{i} \subset D$ innermost with respect to the boundary of $D$ if $\partial D_{i}$ is made up of an $\operatorname{arc} \alpha_{i} \subset D \cap A$ and an arc $\beta_{i} \subset \partial D$ with $D_{i} \cap A=\partial D_{i} \cap A=\alpha_{i}$. Clearly $D$ contains such a disk.

If $\alpha_{\mathrm{i}}$ has both endpoints on one component of $\partial A$, then we can find a disk $D_{i}^{\prime} \subset A$ with $\alpha_{i} \subset \partial D_{i}^{\prime}$ and $\partial D_{i}^{\prime} \subset \partial A \cup \alpha_{i}$. Then $D_{i} \cup D_{i}^{\prime}$ is also a disk. Whether $D_{i} \subset M$ or $D_{i} \subset T$, the usual arguments allow us to isotope $D$ to a new disk in $M \cup T$. with fewer arc intersections.

If $\alpha_{i}$ has its endpoints on opposite components of $\partial A$ and $D_{i} \subset M$, then $A$ must cross a disk in $\partial M$ only once. This is certainly a contradiction. For the same reason we must have that if $D_{i} \subset T, D_{i}$ must be a meridional disk. But then $A$ must run transversely to a meridional disk of $T$. This means that $M \cup T$ is homeomorphic to $M$. But this is a contradiction since $M$ has incompressible boundary.

LEMMA 3.3. $H_{1}\left(M_{i}\right) \simeq \mathbf{Z} \oplus \mathbf{Z}_{d}$ where $d$ is the greatest common divisor of $q_{i}$ and $x$.

COROLlaRY 3.4. Let $M$ be a 3-manifold obtained by $a\left(p_{i}, q_{i}\right)$ surgery along an $(x, y)$ cable knot where $q_{i}$ and $x$ are not relatively prime. Then $M$ is not a homology sphere.

The next theorem seems to be a "folklore" result. It can be deduced from the work of Louise Moser in [5].

THEOREM 3.5. If $a(p, q)$ surgery along a torus knot of type $(x, y)$ yields a homology sphere with finite fundamental group and $q \neq 1$, then $x, y$, and $q$ must be chosen from the numbers 2,3 , and 5 without repetition.

THEOREM 3.6. If $M$ is a manifold obtained by surgery on a link containing a 
sublink which cables about the knot $k$, and surgery on at least two components of the cable sublink cannot be replaced by surgery on $k$, then $M$ is not simply connected.

Proof. Assume that the theorem is false and that $M$ is simply connected. Let $T$ be a regular neighborhood of $k$ such that $\partial T$ contains the cable sublink $\left\{J_{1}, \ldots, J_{n}\right\}$. We assume that each $J_{i}$ curve follows an $(x, y)$ curve on $\partial T$ and that $T_{i}$ is a regular neighborhood of $J_{i}$ with $T_{i} \cap T$ an annulus.

Evidently, if $\left(p_{i}, q_{i}\right)$ surgery is performed on $J_{i}$, we must have $x>1, q_{i}>1$, and $q_{j}>1$ for some $j \neq i$. If this were not the case, surgery on all but possibly one of the components of the cable sublink could be replaced by surgery on $k$. Let $M^{\prime}=T \cup h\left(T_{1}\right) \cup \cdots \cup h\left(T_{n}\right)$ where $h\left(T_{i}\right)$ is the image of $T_{i}$ in $M$ after surgery. $T$ and $h\left(T_{k}\right)$ cannot be attached along a meridional annulus as this would result in $M$ being the connected sum of a lens space and some other manifold.

By Theorem 3.1 and Proposition 3.2 we know that $M^{\prime}$ is irreducible and has incompressible boundary. If $M$ were simply connected, we could by the Dehn lemma [6] and the loop theorem [8], find a real disk $D$ with $\partial D \subset \partial M^{\prime}$ and $D \cap M^{\prime}=\partial D \not 0$ on $\partial M^{\prime}$.

Let $N(D)$ be a regular neighborhood of $D$ in $M$. Then $\operatorname{cl}\left(M-\left(M^{\prime} \cup\right.\right.$ $N(D))$ ) is a homotopy 3-cell. Evidently the surgery on the rest of the components of $L$ has made $\operatorname{cl}\left(M-M^{\prime}\right)$ a homotopy solid torus. We can replace this homotopy 3-cell with a real 3-cell $C$ and obtain a new homology sphere $M^{\prime \prime}$. We note that $C \cup N(D)$ is a solid torus and that $\pi_{1}(M) \simeq$ $\pi_{1}\left(M^{\prime \prime}\right)$. Let $T^{\prime}=C \cup N(D)$.

We write $M^{\prime \prime}$ as $T \cup h\left(T_{1}\right) \cup \cdots \cup h\left(T_{n}\right) \cup T^{\prime}$. We note that $T$ and $T^{\prime}$ are solid tori which intersect along $n$ disjoint nonseparating annuli following an $(x, y)$ curve on $\partial T$. Since $T \cup T^{\prime}$ is embedded in a simply connected manifold, namely $M$, we know by [1] or [2] that $T \cup T^{\prime}$ is a cube-with-torusknotted holes. Hence the nonseparating annuli of sewing between $T$ and $T^{\prime}$ follow $(y, x)$ curves on $\partial T^{\prime}$.

We use Van Kampen's theorem to find a presentation of $\pi_{1}\left(M^{\prime \prime}\right)$. By setting all generators equal to the identity element except the generators of $\pi_{1}(T), \pi_{1}\left(h\left(T_{i}\right)\right)$, and $\pi_{1}\left(T^{\prime}\right)$, we obtain a polyhedral group as a homomorph of $\pi_{1}\left(M^{\prime \prime}\right)$. As in the proof of Theorem 3.5, we compare those polyhedral groups which are finite with those whose abelianization is trivial and find that $x, y$, and $q_{i}$ must be chosen from the numbers 2,3 , and 5 without repetition. Likewise $x, y$, and $q_{j}$ must be chosen from the numbers 2,3 , and 5 without repetition. This means that $q_{i}=q_{j}$.

We can find a nonsingular annulus $A \subset T$ which is transverse to the meridional disks of $T$ and one of whose boundary components lies in the annulus of sewing between $h\left(T_{i}\right)$ and $T$ and the other in the annulus of sewing between $h\left(T_{j}\right)$ and $T$. Then if $N(A)$ is a regular neighborhood of $A$ in $T$, then $h\left(T_{i}\right) \cup N(A) \cup h\left(T_{j}\right)$ will be a manifold which by Lemma 3.3 has 


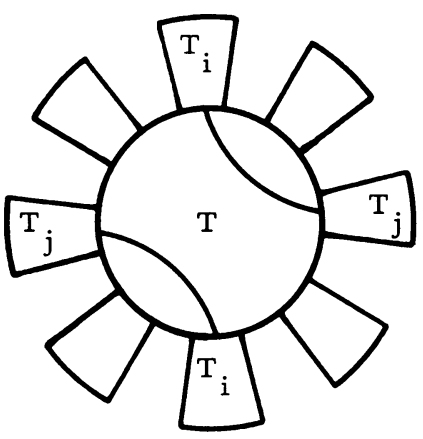

FIGURE 2

1st homology $\mathbf{Z} \oplus \mathbf{Z}_{d}$. In fact, $d=q_{i}=q_{j}>1$. Therefore, by a simple Mayer-Vietoris argument, $M^{\prime \prime}$ is not a homology sphere which is a contradiction.

\section{REFERENCES}

1. Bradd Clark, Knot theories of the lens spaces, Ph.D. Thesis, University of Wyoming, 1976.

2. Wolfgang Heil, Elementary surgery on Seifert fiber spaces, Yokohama Math. J. 22 (1976), 135-139.

3. John Hemple, A simply connected 3-manifold is $S^{3}$ if it is the sum of a solid torus and the complement of a torus knot, Proc. Amer. Math. Soc. 15 (1964), 154-158.

4. W. B. R. Lickorish, A representation of orientable combinatorial 3-manifolds, Ann. of Math. (2) 76 (1962), 531-540.

5. Louise Moser, Elementary surgery along torus knots and solvable fundamental groups of closed 3-manifolds, Ph.D. Thesis, University of Wisconsin, 1970.

6. C. D. Papakyriakopoulos, On Dehn's lemma and the asphericity of knots, Ann. of Math. (2) 66 (1957), 1-26.

7. Jonathan Simon, Some classes of knots with property P, Topology of Manifolds, edited by James Cantrell and C. H. Edwards, Jr., Markham, Chicago, Ill., 1970.

8. J. Stallings, On the loop theorem, Ann. of Math. (2) 72 (1960), 12-19.

Department of MATHEMatics, UniverstTy of SOUTHWESTERn Louisiana, LAFAYeTte, LoulSIANA 70504 